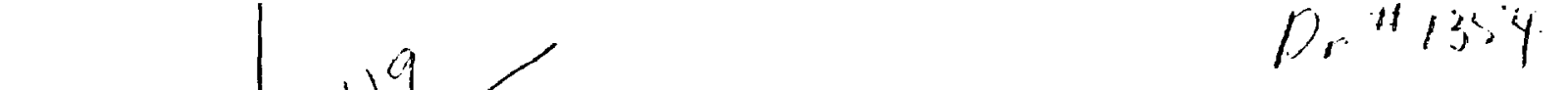

LA-6748-MS

Informal Report

Issued: IMay 1977

\title{
The LAMPF Line D Fast Deflector System
}

\author{
by
}

R. K. Cooper

J. R. Faulkner *

"EIMAC Division of VARIAN Associates, San Carlos, California.

lOS alamos, NEW MEXICO 87545

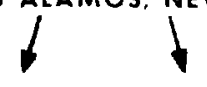

An Aflirmative Action / Equal Opportunity Employer 
THE LAM PF LINE D FAST DEFJ ECTOR SYSTEM

by

\author{
R. K. Cooper and J. R. Faulkner
}

\begin{abstract}
The design, fabrication, and testing of a fast kicker system for $800-\mathrm{MeV}$ protons is described. The kicker magnet current pulse has a $1-\mu \mathrm{s}$ rise time, with a 10- $\mu \mathrm{s}$ flat top. The ferrite magnet is $1.09 \mathrm{~m}$ long, has a field strength of $1.25 \mathrm{kG}$, and is pulsed at up to $7000 \mathrm{~A}, 120 \mathrm{~Hz}$ by a 2-ohm, six-section, lumped-element pulse-forming network.
\end{abstract}

\section{INTRODUCTION}

The Weapons Neutron Research Facility is only one of many experimental areas that use the 800 $\mathrm{MeV}$ proton beam from the Clinton P. Anderson Meson Physics Facility (LAMPF). Designated Line $D$, this area may use entire $500-\mu$-wide beam pulses at pulse rates up to $12 \mathrm{~Hz}$ or may choose to take the last $5 \mu \mathrm{s}$ off each pulse at a $120-\mathrm{Hz}$ rate. This report describes the deflection system used to accommodate the latter mode of operation.

The proton beam is deflected into Line $D$ in a 1.09-m-long, 1.25-kG, ferrite magnet, pulsed at up to $7000 \mathrm{~A}, 120 \mathrm{~Hz}$ by a $2-\mathrm{ohm}$, six-section, lumpedelement, pulse-forming network (pfn). The surrent pulse into the magnet has a rise and fall time of about $1 \mu \mathrm{s}$ and a $9-\mu \mathrm{s}$ usable flat top portion. This line is tuned to produce a current pulse with $\pm 0.2 \%$ ripple across the flat top portion. The isw-ripple current is necessary to minimize jitter as the beam enters line D.

\section{PROTOTYPE DESIGN VALUES}

Pulse lines of the sort used in the Line $\mathbb{D}$ fast deflection system are basically lumped-element ap- proximations to a finite length of charged transmission line discharging into a matched load. The characteristic impedance of a transmission line is given by $Z_{0}=\sqrt{L / C}$, where $L$ and $C$ are. respectively, the inductance and capacitance per unit length, and the speed of waves on the line is $\mathrm{v}=$ $1 / \sqrt{\mathrm{LC}}$, so that the discharge current pulse duration, $\tau$, is $2 \ell \sqrt{\mathrm{LC}}$, where $\ell$ is the line length. Pulse lines are frequently designed as $\mathrm{N}$ identical L-C ladder sections (Fig. 1) with lumped inductance and capacitance values $\left(\mathrm{L}_{\ell}, \mathrm{C}_{\ell}\right)$ that satisfy

$$
\sqrt{\frac{\mathrm{L}_{Q}}{\mathrm{C}_{\ell^{\prime}}}}=\mathrm{Z}_{0} \text { and } \sqrt{\mathrm{L}_{Q} \mathrm{C}_{Q}}=\frac{\tau}{2 \mathrm{~N}} \text {, }
$$

where $\tau$ is the appropriate pulse length and $Z_{0}$ is the circuit impedance level, which is fixed if $\tau$ and $L_{\ell}$ are fixed.

Pulse lines designed according to Eqs. (1) produce the load currents shown in Fig. 2 for $N=1$ to 7 . The initial overshoot is clearly associated with the first section, as it is present in the waveform for $\mathrm{N}=1$. This overshoot occurs because at $t=0+$, just after the switch is closed, the full voltage applied to the capacitors appears across the first inductor, whereas in the transmission line only half the charging 


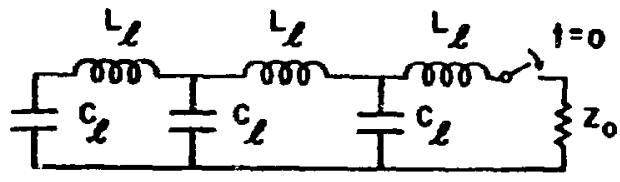

Fig. 1.

Conventional pulse line.

voltage appears at the terminals after the switch is closed.

For our kicker magnet, we wanted to eliminate ihe overshoot and make as uniform a current pulse as possible, to minimize current regulation requirements. Accordirifly, we modeled the pulse line on an EAI-280 a 1 alog computer and adjusted the inductance and capacilance of each section of the lire to achieve the curres st waveforms shown in Fig. 3. The analog circuit for $N=3$ is shown in Fig. 4. Table $I$ summarizes the empirically determined values of $\mathrm{L}$ and $\mathrm{C}$ that produce the waveforms shown in Fig. 3. The table presents normalized values; they are appropriate to a 1-ohm load and produce a 1-s pulse. To produce a pulse of $\tau$ seconds and an impedance level of $Z_{0}$ ohms, the table entries should be scaled according to

$L_{\text {actual }}=\tau \mathrm{Z}_{0} \mathrm{~L}_{\mathrm{tab} \text { le }}$ and $\mathrm{C}_{\mathrm{actual}}=\frac{\tau}{\mathrm{Z}_{0}} \mathrm{C}_{\mathrm{tablc}}$.

The Line $\mathrm{D}$ kicker magnet has a nominal design inductance of $2.67 \mu \mathrm{H}$. We wanted a $12.5-\mu \mathrm{s}$ pulse, so the impedance level of the line was determined to be $Z_{0}=L_{1, \text { table }} / \tau \mathrm{L}_{\text {magnet }}=1.99 \Omega$. The pulse line was then designed using the nominal vulues given in Table I for $\mathbf{N}=6$.

\section{CONSTRUCTION DETAILS}

Figure 5 shows the pulse network to be a stack of coaxial capacitor sections with the inductors connected in series down the center of the stack. Each section is double-walled and water-cooled, and the interior of the entire stack and the thyratron switch are forced-air cooled. Wach capacitor in the upper level of each of the six sections is connected to the section above it by an individual plug. The inductors are connected in the same way so that the stack may be broken and lifted apart at any joint without removing any capacitors or inductors. One need only remove the screws in the outer flange at the point where the stack is to be broken and lift the line apart.

This coaxial construction provides a very low inductance conrection for the components. It also provides mutual inductance shielding for the inductors, so all the $\mathrm{L}$ and $\mathrm{C}$ may be assumed to be in the lumped elements.

At original assembly, the six capacitor sections were set to the values indicated by the analog computer simulation. These values were adjusted by using $0.05-, 0.02-, 0.01-$, and $0.001-\mathrm{pF}$ capacitor

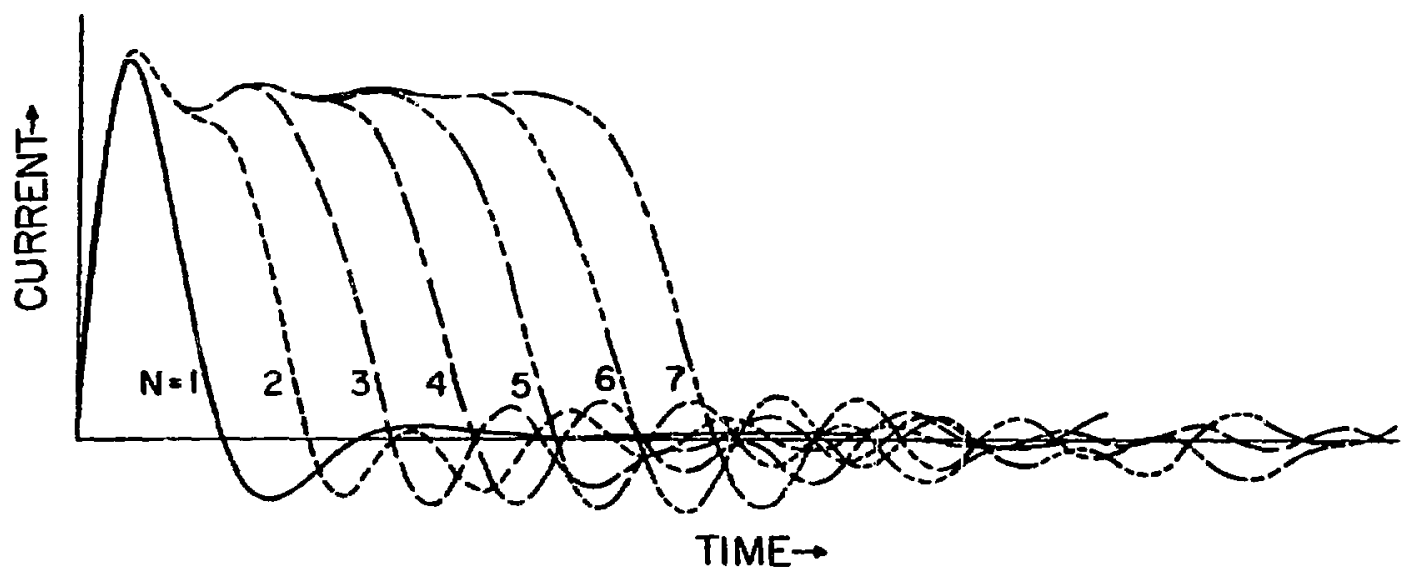

Fig. 2.

Load currents produced by con'ontional pulse lines. 


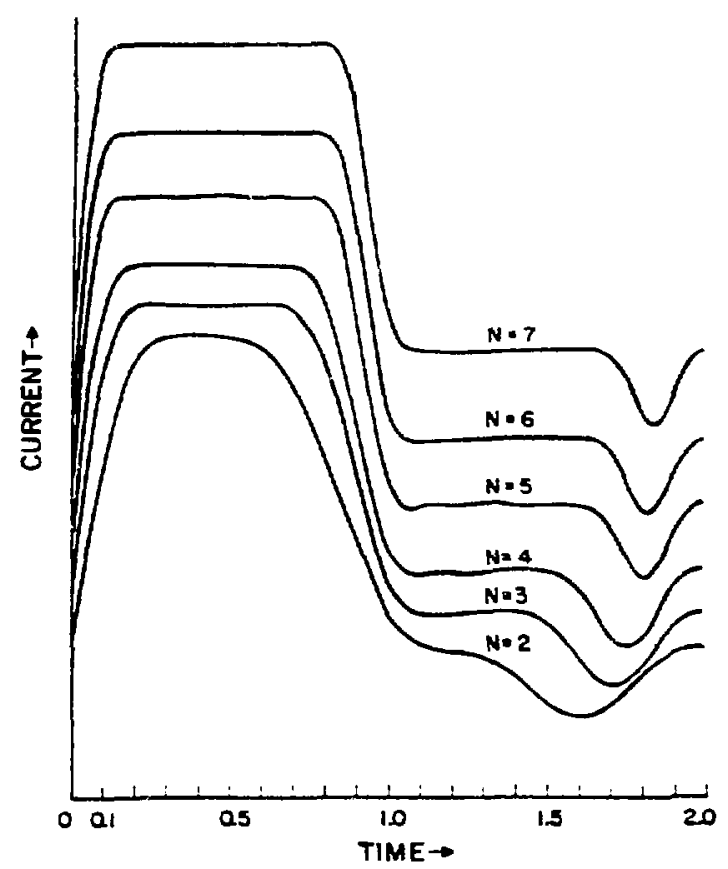

Fig. 3.

Load currents produced by analog-modeled pulse lines.

values. The capacities were measured using a digital capacitance meter and adjusted to three-place accuracy. We did not feel that the inductor values could be measured with the same accuracy as those of the capacitors, so the capacitances were set accurately and the inductors were then adjusted to give the pulse shape we wanted. The computer simulation was used at each step of inductor tuning to predict the next step. After final tuning, the inductance values were measured as accurately as the equipment on hand would permit and were found to be almost exactly the values predicted by the original computer simulation. The resultant pulse shape was exactly as shown by simulation.

One of the main areas of concern in this high peak, high average power application was the design of the $2-\Omega$ load resistor. Several different configurations were considered. The temperature control and liquid-handling involved in using a salt solution were thought to be too complicated, and it is difficult to maintain good electrical contact with glo-

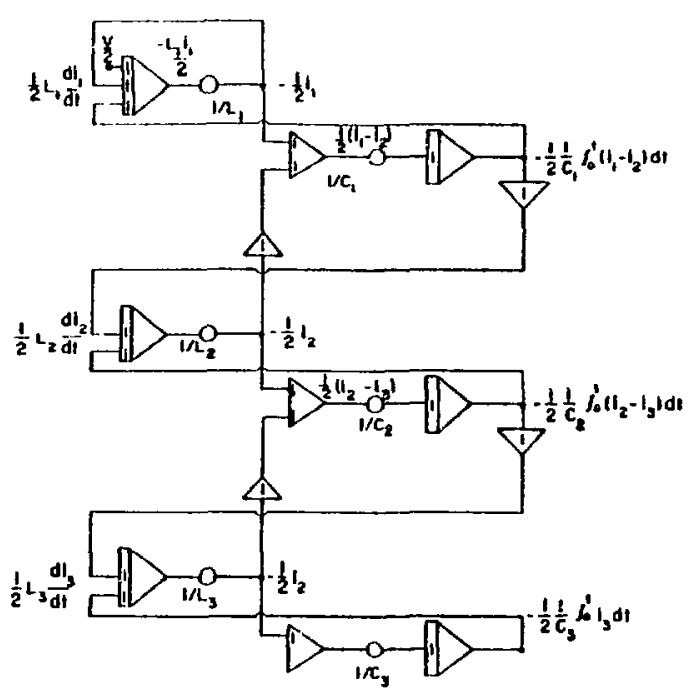

Fig. 4.

Analog computer circuit for modeling a threesection pulse line.

bar resistors. A suitable low-inductance, highpower, $100-\mathrm{kW}$ at $0.25 \mu \mathrm{H}$, ohm-weave resistor could be constructed, but it would be expensive, large, and difficult to maintain. Such resistors were tested, and the pulse waveform was not degraded, but we felt that a cheaper, simpler unit could be made if some rise-time degradation could be tolerated. The final load resistor is composed of sections of very thin-walled stainless steel tubing immersed in a cooling water jacket. The $2.98-\mu \mathrm{H}$ inductance of this load resistor does degrade the pulse. rise time, but subsequent adjustment of $\mathrm{C}_{1}, \mathrm{C}_{2}$, and $\mathrm{C}_{s}$ gives a pulse with a rise time of $3 \mu \mathrm{s}$ and a $6.5-\mu \mathrm{s}$ flat top. The current ripple on the flat top is $\pm 0.2 \%$. This unit is very compact, inexpensive, and easily replaceable.

\section{SYSTEM DESCRIPTION}

The magnet pulser used for the line D fast deflector system is shown in Fig. 6. Although its power consumption is not the most efficient, this system has many features that provide very attractive flexibility. The added power dissipation of the hardtube charge control system makes the average power 
TABLE I

PULSE-F SRMING NETWORK ELEMENT VALUES

\begin{tabular}{|c|c|c|c|c|c|c|c|c|}
\hline \multirow{2}{*}{$\begin{array}{l}\text { No. of } \\
\text { Sections }\end{array}$} & & \multicolumn{7}{|c|}{$\mathbf{i}$} \\
\hline & & 1 & 2 & 3 & 4 & 5 & 6 & 7 \\
\hline \multirow[t]{2}{*}{2} & $\mathbf{L}_{1}$ & 0.2559 & 0.1388 & & & & & \\
\hline & $\mathrm{C}_{\mathrm{s}}$ & 0.1444 & 0.1785 & & & & & \\
\hline \multirow[t]{2}{*}{3} & $\mathrm{~L}_{1}$ & 0.1817 & 0.0945 & 0.1172 & & & & \\
\hline & $\mathrm{C}_{1}$ & 0.1024 & 0.0991 & 0.1733 & & & & \\
\hline \multirow[t]{2}{*}{4} & $\mathrm{~L}_{1}$ & 0.1503 & 0.0721 & 0.0775 & 0.1023 & & & \\
\hline & $C_{1}$ & 0.0818 & 0.0744 & 0.0851 & 0.1517 & & & \\
\hline \multirow[t]{2}{*}{5} & $\mathbf{L}_{1}$ & 0.1145 & 0.0581 & 0.061 .3 & 0.0691 & 0.0968 & & \\
\hline & $C_{1}$ & 0.0647 & 0.0597 & 0.0662 & 0.0806 & 0.1488 & & \\
\hline \multirow[t]{2}{*}{6} & $\mathrm{~L}$ & 0.1062 & 0.0489 & 0.0491 & 0.0535 & 0.0624 & 0.0878 & \\
\hline & $\mathrm{C}_{2}$ & 0.0579 & 0.0484 & 0.0521 & 0.0589 & 0.0727 & 0.1378 & \\
\hline \multirow[t]{2}{*}{7} & $\mathbf{L}_{1}$ & 0.1016 & 0.0445 & 0.0429 & 0.0429 & 0.0486 & 0.0571 & 0.0811 \\
\hline & $\mathrm{C}_{1}$ & 0.0521 & 0.0442 & 0.0432 & 0.0456 & 0.0531 & 0.0659 & 0.1261 \\
\hline
\end{tabular}

consumption essentially double that of a conventional resonant charge system. However, use of the EIMAC 4CW 100,000 D hard-inbe system allows delayed charge, fault iaterruption, charge programming on a pulse-to-pulse basis, $\varepsilon$ nd closed-loop control over the charge on the pulse line. This flexibility, in a system that must operate with the overall LAMPF facility and cause minimal interference, makes the added power consumption a small price to pay for the operaticnal ease provided.

The control and firing system operates as follows. At initial turn-on, the hard tube in the charge control loop is biased off, so no charge is applied to the pulse-forming network (pfn). The first firing pulse from the accelerator control system enables the charge control loop after a preset charge delay but does not fire the thyratron, as the charge-OK signal is not at the proper logic level. After the charge delay, the enable signal sent to the hard tube modulater (HTM) pulses the bias to zero and the screen grid to $2000 \mathrm{~V}$, allowing charging current to flow into the pfn. When the pfn voltage reaches a level preset by the control potentiometer, the charge control signal is removed, thereby turning off the switch tube by restoring the $500-\mathrm{V}$ bias and removing the 2000-V screen grid signal. Then, the logic system sees a charge enable signal and zero output from the charge control system, indicating that the loop is active and the line charge is proper. The thyratron may then fire on the next firing pulse from the main accelerator control system. For this initial pulse, the entire charge on the pfn is accomplished during a relatively short interval between charge enable and the firing pulse; this requires very high peak power from the switch tube and would severely shorten switch tube life. To maximize tube life by minimizing peak power iequirements while still supplying the required sverage power, all succeeding pulses are charged differently, as follows.

The firing of the thyratron and the resulting voltage drop at point $A$ are coupled to the switch tube bias flip-flop by $C_{1}$, thus removing the bias from $V_{1}$. With zero bias and zero screen voltage, the switch tube then begins to conduct as a diode and starts to recharge the pulse line at a very low current 


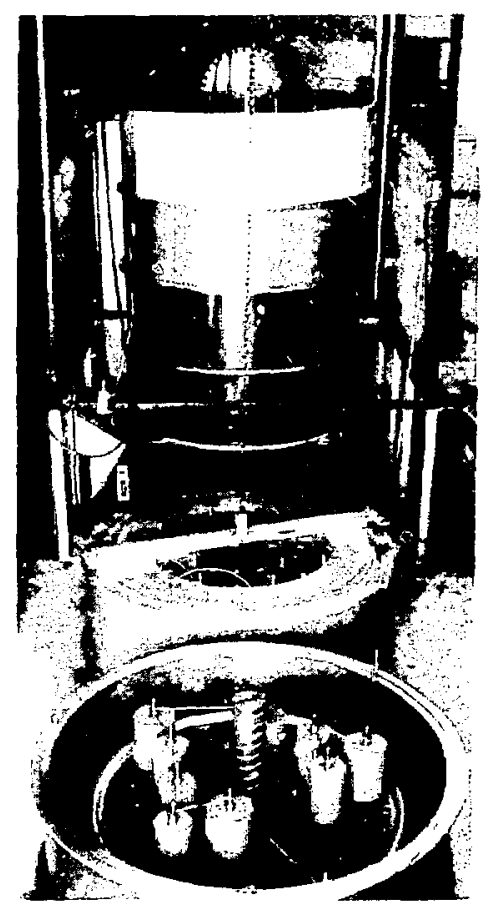

Fig. 5.

The pulse-forming netuork as fabricated.

until the charge delay time elapses. The charge control system then applies $2000 \mathrm{~V}$ to the HTM screen grid to raise the line charge to the proper level. When the pfn reaches proper charge level, the 2000 . $V$ screen signal is removed and the bias is restored to the HTM. The line is now charged, and the charge control system is turned off, awaiting the next firing pulse. By choosing $\mathrm{L}_{1}$ so that the "free" charge time through $V_{1}$ is long compared to the pulse interval and by delaying the closed loop charge-enable signal to allow maximum free charge, one minimizes the high peak current or demand charge interval. This reduces wear and tear on the swich tubes.

As discussed, the thyratron is not fired unless the line charge is proper. Inputs to the kicker system logic also allow for fast inhibition of firing in the event of other accelerator problems. The thyratron current is monitored by the logic system and compared on a pulse-to-pulse basis with the firing signal from the accelerator control timer. If the two do not coincide exactly, the charge and firing systems are inhibited and a digital counter is advanced one count, indicating a misfire. After such a count, the

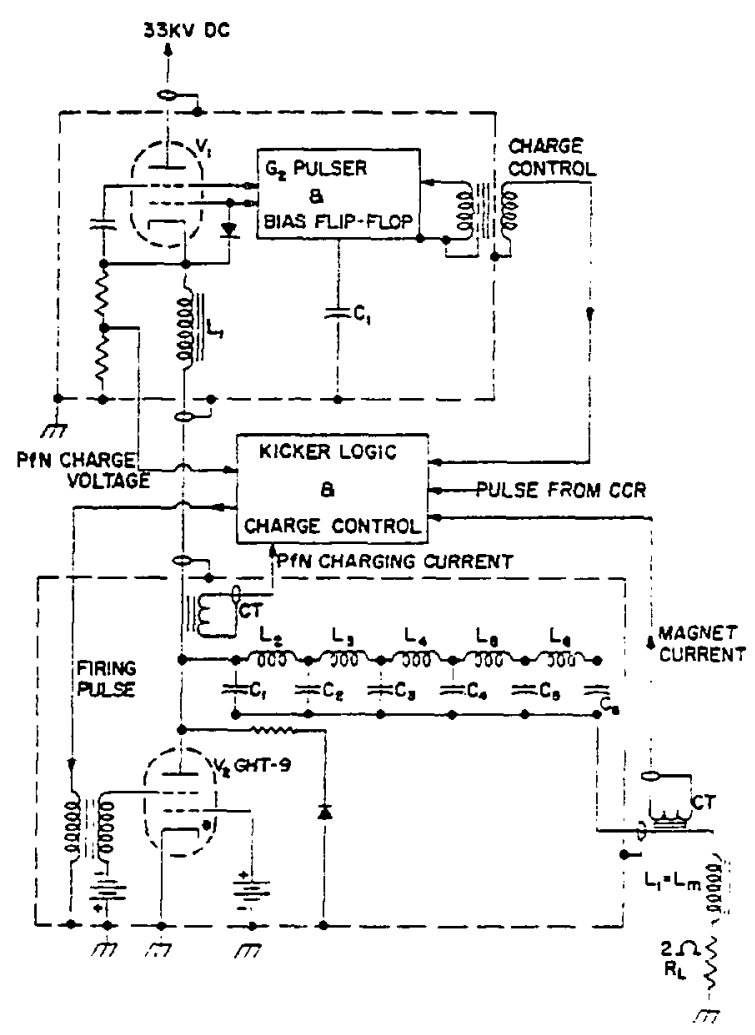

Fig. 6.

The magnet pulser.

previously described charging sequence must start again. In the event of 10 such misfires, the logic locks out the charge system until it is checked and reset, either by computer or by the accelerator operator.

Another feature of this control system is that it can be programmed to different current outputs on a pulse-to-pulse basis. An analog signnal may be used in place of the set point potentiometer and stepped to different voltages on a pulse-to-pulse basis, which would allow kicking to different beam lines, thus greatly diversifying the experimental area capabilities.

\section{CONCLUSION}

This system has been tested extensively at full power. A total of $10^{8}$ pulses has been fired at the 120 $\mathrm{Hz}$ rate. During several 8 - to 12 -h runs, capacitor overheating was the only significant problem. After 
several hours running, some of the $0.05-\mu \mathrm{F}$ capacitors became overheated and several exploded. No other size of capacitors heated excessively or failed. The $0.05 \mu \mathrm{F}$ unit has been redesigned to provide more plate area, better heat flow from the interior of the capacitor to the cooled tank walls, and higher temperature processing. The new design should eliminate capacitor overheating as a major problem.

Although the peak current magnitude is similar to that in other accelerator applicatione and the 120 -
$\mathrm{Hz}$ rate is much higher, the usual kicker requirement is for pulses whose rise time is much faster than the 1-3 $\mu$ s used here. The same simulation and basic construction techniques are entirely applicable but, certainly, a load resistor with extremely low inductance would be required to give the faster pulse rise times. However, most such applications will not require the load resistor to handle the $100 \mathrm{~kW}$ of average power, so the load resistor should be easier to design. 